\title{
Role of Lipid Peroxidation in Gastric Mucosal Lesions Induced by Ischemia-Reperfusion in the Pylorus-Ligated Rat
}

\author{
Junko TANAKA* and Yasukatsu YUDA \\ Pharmaceutical Research Center, Meiji Seika Kaisha, Ltd., Drug Research Laboratories, Morooka-cho, Kohoku-ku, \\ Yokohama 222, Japan. Received March 18, 1992
}

The peroxidation of lipids and changes in the activities of related enzymes, such as xanthine-xanthine oxidase (XOD), superoxide dismutase (SOD), and glutathione peroxidase (GSH-px) in the gastric mucosa were studied in rat model of ischemia-reperfusion with pylorus ligation. Myeloperoxidase (MPO), a marker enzyme of leucocytes, was also studied. Thiobarbituric acid reactive substances (TBA RS) in gastric mucosa were significantly increased by clamping the celiac artery for $30 \mathrm{~min}$ and reperfusion for $60 \mathrm{~min}$ after $3 \mathrm{~h}$ of pylorus ligation. XOD activity in gastric mucosa increased with the developmeat of gastric mucosal injury. Allopurinol significantly suppressed XOD activity but did not inhibit mucosal injury or the increase in TBA RS. MPO activity in the gastric mucosa was significantly increased by gastric mucosal injury. Famotidine significantly inhibited the increase in MPO activity in gastric mucosa, while allopurinol did not. SOD and GSH-px activities in the gastric mucosa were decreased significantly by gastric mucosal injury. SOD activity was normal following treatment with famotidine and allopurinol. Moreover, GSH-px activity recovered to the normal level with famotidine and allopurinol treatment. These findings suggest that oxygen radicals and lipid peroxidation can cause gastric mucosal injury by ischemia-reperfusion in the pylorus-ligated rat. The generation of oxygen free radicals may be derived mainly from activated polymorphonuclear leukocytes (PMN), and the decrease in SOD and GSH-px activity in gastric mucosa seems to aggravate mucosal injury by free radicals and lipid peroxidation.

Keywords ischemia-reperfusion; lipid peroxidation; polymorphonuclear leukocyte; oxygen free radical; xanthine-xanthine oxidase; gastric ulcer

\section{Introduction}

It has been reported that oxygen free radicals play an important role in the pathogenesis of tissue injury induced by ischemia of the small intestine, ${ }^{1)}$ heart, ${ }^{2)}$ kidney, ${ }^{3)}$ liver, ${ }^{4)}$ and skin. ${ }^{5)}$ In gastric injury, it has been suggested that ischemia-reperfusion and hemorrhagic shock may be caused by these free radicals as well. ${ }^{6-8)}$ Most evidence for a role of oxygen radicals in the pathogenesis of acute gastric mucosal lesion has been obtained indirectly by using oxygen radical scavenging agents such as superoxide dismutase (SOD) and dimethyl sulfoxide (DMSO). Ischemia and the presence of acid in the stomach may produce severe gastric mucosal damage. Furthermore, oxygen free radicals produced by ischemia-reperfusion can attack and initiate a free radical chain reaction known as lipid peroxidation. ${ }^{9)}$ These pathological changes may cause acid and pepsin to attack the gastric mucosa, producing erosion or an ulcer. The major sources of active oxygen species produced after ischemia-reperfusion seem to be the enzyme xanthine-xanthine oxidase (XOD) ${ }^{6-8)}$ and activated polymorphonuclear leukocytes $(\mathrm{PMN}) .^{10,11)}$ In this study, we measured thiobarbituric acid reactive substances (TBA RS), XOD, and myeloperoxidase (MPO) in the gastric mucosa in order to elucidate the mechanism of oxygen radical generation and the pathogenesis of gastric mucosal injury during ischemia and reperfusion in the pylorus ligated rat. Furthermore, we measured the levels of SOD (superoxide radical scavenger), and glutathione peroxidase (GSH-px) (an enzyme that protects animal cells against peroxidative damage ${ }^{12)}$ ) in the gastric mucosa in order to elucidate the productive and resolvent systems of lipid peroxidation against tissue peroxidative damage.

\section{Experimental}

Animals Male Donryu rats (SPF), 8 weeks old and weighing 200 $230 \mathrm{~g}$, were used. The animals were obtained from Charles River Co., Tokyo.

Materials Allopurinol was obtained from Fukuju Pharmaceuticals, Ltd. Famotidine was produced by our company. Thiobarbituric acid (TBA) was obtained from BHD Chemicals, Ltd. Phosphotungstic acid was obtained from Merck, West Germany. Glutathione (GSH) reductase, GSH-px standard solution and xanthine oxidase solution were obtained from Boehringer Mannheim Yamanouchi, Tokyo. Hydroxylamine hydrochloride, sulfanic acid, and the uric acid test kit were obtained from Wako Pure Chemical Industries, Ltd., Tokyo. N-(1-Naphthyl)ethylenediamine dihydrochloride was obtained from Tokyo Kasei, Tokyo. $\beta$-Nicotinamide adenine dinucleotide phosphate was obtained from Kohjin Co., Ltd., Tokyo. GSH and $o$-dianisidine dihydrochloride were obtained from Sigma, U.S.A. The other chemicals were of reagent grade and were used without purification.

Experimental Ulcers Experimental ulcers were produced according to the modified method of Yosikawa and his coworkers. ${ }^{13)}$ Drugs were administered per os to rats once a day for $3 \mathrm{~d}$. Rats previously fasted for $18-24 \mathrm{~h}$ were anesthetized with pentobarbital sodium, and anesthesia was maintained with ether. The pylorus of the rats was. ligated $1 \mathrm{~h}$ after the last administration of the drugs. After $3 \mathrm{~h}$, the celiac artery was clamped for $30 \mathrm{~min}$ and then reperfused for $1 \mathrm{~h}$. The rats were sacrificed and the stomach of each rat was removed. The collected stomach mucous membranes were frozen at $-90^{\circ} \mathrm{C}$ until use.

Determination of Lipid Peroxidation The level of TBA RS in the gastric mucosa was measured according to the method of Ohkawa et al. ${ }^{14)}$ The gastric mucosa was homogenized with $1.15 \% \mathrm{KCl}$ solution to obtain a $10 \%$ homogenate solution. After adding sodium dodecyl sulfate and TBA solution, the reaction product was assayed spectrophotometrically $(532 \mathrm{~nm})$.

Measurement of XOD XOD-catalyzed uric acid formation in the gastric mucosa was monitored spectrophotometrically at $550 \mathrm{~nm}$. The reaction mixture consisted of $100 \mathrm{~mm}$ of a phosphate-boric acid buffer $(\mathrm{pH} 8.2)(0.3 \mathrm{ml})$, xanthine $(0.2 \mathrm{ml})$, distilled water $(0.3 \mathrm{ml})$ and the sample solution $(0.2 \mathrm{ml})$. A sample of supernatant fluid with $20 \%$ homogenate solution and $1.15 \% \mathrm{KCl}$ was made by centrifugation at $4000 \times g$ for $10 \mathrm{~min}$ at $4^{\circ} \mathrm{C}$. The level of uric acid in the reaction mixture was measured by the Wako uric acid test kit after incubation for $3 \mathrm{~h}$ at $37^{\circ} \mathrm{C}$

Measurement of MPO MPO activity was determined according to 
the modified method of Bradley and his coworkers. ${ }^{15)}$ The reaction mixture consisted of $50 \mathrm{~mm}$ of a phosphate buffer (pH 6), $1.03 \mathrm{~mm}$ $o$-dianisidine and $1 \% \mathrm{H}_{2} \mathrm{O}_{2}$ in a total volume of $180 \mu \mathrm{l}$. Samples $(20 \mu \mathrm{l})$ were added to the above mixture, and absorbance at $450 \mathrm{~nm}$ was immediately recorded for $5 \mathrm{~min}$ with a microplate reader. A sample of the supernatant fiuid with $10 \%$ homogenate solution and $1.15 \% \mathrm{KCl}$ was made by centrifugation at $4000 \times g$ for $10 \mathrm{~min}$ at $4{ }^{\circ} \mathrm{C}$. Activity was calculated from optical density (OD) per min.

Measurement of SOD SOD activity in the gastric mucosa was assayed by a modification of the Nitrite kit method utilized for the xanthine oxidase-naphthyl ethylenediamine system. ${ }^{16)}$ Optical absorption was measured at $550 \mathrm{~nm}$. The reaction mixture consisted of $100 \mathrm{~mm}$ of a phosphate-boric acid buffer (pH 8.2) $(0.2 \mathrm{ml}), 5 \mathrm{mM}$ xanthine $(0.2 \mathrm{ml})$, $1 \mathrm{~mm}$ hydroxylamine $\mathrm{HCl}(0.1 \mathrm{ml})$, distilled water $(0.1 \mathrm{ml})$, xanthine oxidase solution $(0.1 \mathrm{U} / \mathrm{ml})$, and sample solution $(0.1 \mathrm{ml})$. A sample of the supernatant fluid with $10 \%$ homogenate solution and $1.15 \% \mathrm{KCl}$ was made by centrifugation at $4000 \times g$ for $10 \mathrm{~min}$ at $4^{\circ} \mathrm{C}$.

Measurement of GSH-px GSH-px activity was determined according to the method of Lawrence et al. ${ }^{17)}$ The reaction mixture consisted of $50 \mathrm{~mm}$ potassium phosphate buffer ( $\mathrm{pH}$ 7), $1 \mathrm{~mm}$ EDTA, $1 \mathrm{mM} \mathrm{NaN}_{3}$, $0.2 \mathrm{~mm}$ reduced nicotinamide adenine dinucleotide phosphate (NADPH), 1 E.U./ml oxidized glutathione (GSSG)-reductase, $1 \mathrm{mM} \mathrm{GSH}$, and $0.25 \mathrm{mM} \mathrm{H}_{2} \mathrm{O}_{2}$ in a total volume of $1 \mathrm{ml}$. The ingredients, except the enzyme source and peroxide, were combined at the beginning of each assay. Samples $(0.1 \mathrm{ml})$ were added to $0.8 \mathrm{ml}$ of the above mixture and incubated for $5 \mathrm{~min}$ at $25^{\circ} \mathrm{C}$ before initiating the reaction with the addition of $0.1 \mathrm{ml}$ of peroxide solution. A sample of supernatant fluid with $10 \%$ homogenate solution and $1.15 \% \mathrm{KCl}$ was made by centrifugation at $4000 \times \mathrm{g}$ for $10 \mathrm{~min}$ at $4^{\circ} \mathrm{C}$. The absorbance at $340 \mathrm{~nm}$ was recorded for $5 \mathrm{~min}$. The activity was then calculated from the slope of the lines as $\mu \mathrm{mol}$ of NADPH oxidized per min. The blank datum (the enzyme was replaced with distilled water) was subtraced from each value.

Measurement of Protein The amount of protein was determined by the method of Lowry et al. ${ }^{18)}$

\section{Results}

The total area of gastric mucosal injury increased after clamping the celiac artery for $30 \mathrm{~min}$ and reperfusing it for $60 \mathrm{~min}$ while performing pylorus ligation for $3 \mathrm{~h}$. The extent of gastric mucosal injury was determined by MCID of Imaging Research, Inc. The results are shown in Table I. The change was significantly inhibited by treatment with famotidine, but allopurinol treatment did not inhibit gastric mucosal injury.

The level of TBA RS in the gastric mucosa, an index of lipid peroxidation, increased after clamping the celiac artery for $30 \mathrm{~min}$ and reperfusion for $60 \mathrm{~min}$ while performing pylorus ligation for $3 \mathrm{~h}$. This change was significantly inhibited by treatment with famotidine, but not by allopurinol. The results are shown in Fig. 1. The level of TBA RS in the stomach varied with the extent of gastric mucosal injury. The level of TBA RS in the stomach was correlated to the index of gastric mucosal injury $(r=0.823)$.

As shown in Fig. 2, XOD activity was significantly

TABLE I. Effects of Famotidine and Allopurinol on Gastric Mucosal Injury Index

\begin{tabular}{lcc}
\hline \multicolumn{1}{c}{ Treatment } & $\begin{array}{c}\text { Gastric mucosal injury index } \\
\left(\mathrm{mm}^{2}\right)\end{array}$ \\
\hline Control & & $562.0 \pm 101.6$ \\
Famotidine & $30 \mathrm{mg} / \mathrm{kg}$ & $3.4 \pm 2.2^{a)}$ \\
Allopurinol & $100 \mathrm{mg} / \mathrm{kg}$ & $500.0 \pm 62.7$
\end{tabular}

Gastric mucosal injury index was measured by the total area of erosions induced by ischemia and reperfusion in pylorus ligated rats. Each value is the mean \pm S.D. from 4-5 determinations. Saline solution was used as the control. a) $p<0.001$. increased by clamping the celiac artery for $30 \mathrm{~min}$ and reperfusion for $60 \mathrm{~min}$ while performing pylorus ligation for $3 \mathrm{~h}(p<0.001)$. Treatment with allopurinol significantly decreased XOD activity in the gastric mucosa, whereas

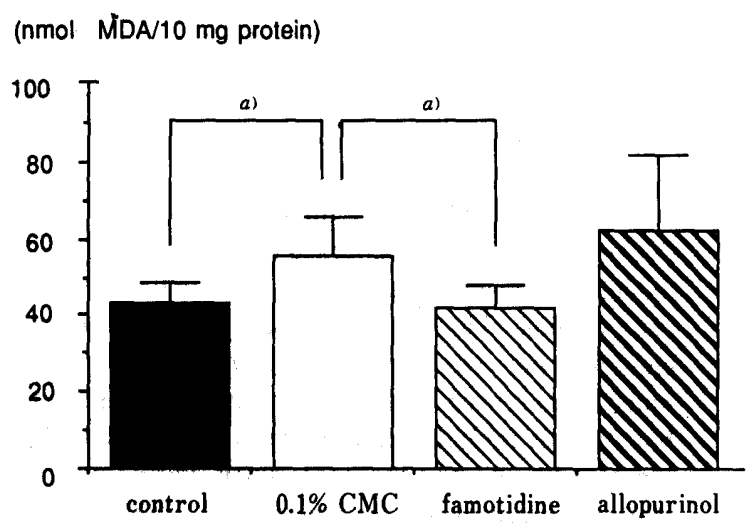

Fig. 1. Effect of Famotidine and Allopurinol on TBA Reactant Substance Level in the Gastric Mucosa Lesions Induced by IschemiaReperfusion in the Pylorus Ligated Rat

TBA reactant substance level in the gastric mucosa after ischemia-reperfusion with pylorus ligation for $3 \mathrm{~h}$ in rats. Each value is the mean \pm S.D. from $7-10$ determinations. An untreated rat was used as the control. a) $p<0.01$.

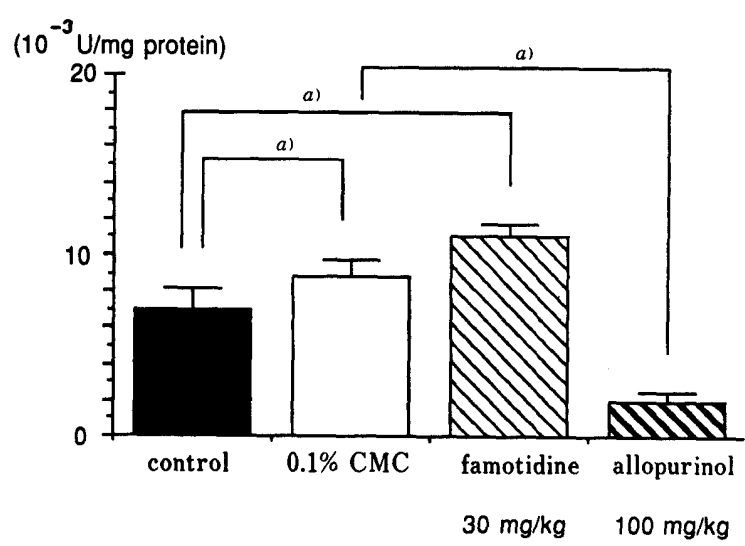

Fig. 2. Effect of Famotidine and Allopurinol on XOD Activity in the Gastric Mucosa Lesions Induced by Ischemia-Reperfusion in the Pylorus Ligated Rat

XOD activity in the gastric mucosa after ischemia-reperfusion with pylorus ligation for $3 \mathrm{~h}$ in the rats. Each value is the mean \pm S.D. from 7-10 determinations. An untreated rat was used as the control. a) $p<0.001$.

\section{(U/mg protein)}

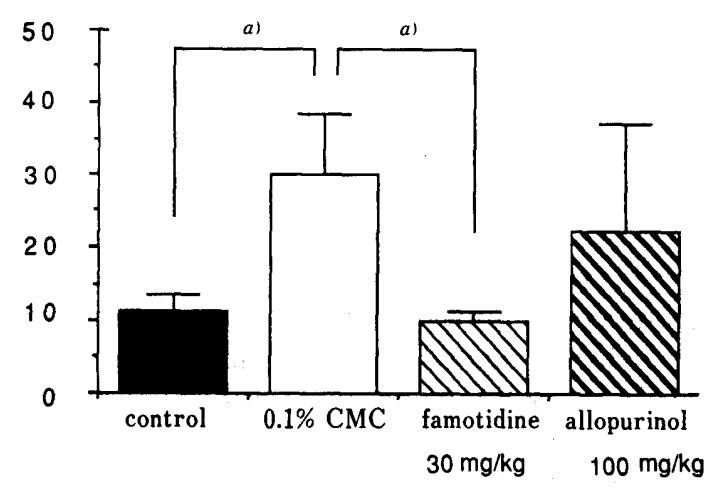

Fig. 3. Effect of Famotidine and Allopurinol on MPO Activity in the Gastric Mucosa Lesions Induced by Ischemia-Reperfusion in the Pylorus Ligated Rat

Levels of MPO activity in the gastric mucosa after ischemia-reperfusion with pylorus ligation for $3 \mathrm{~h}$ in the rats. Each value is the mean \pm S.D. from $7-10$ determinations. a) $p<0.001$. 
famotidine did not inhibit the increase in XOD activity. The level of TBA RS in the stomach did not correlate with XOD activity in the stomach $(r=0.361)$.

Changes in MPO activity are shown in Fig. 3. MPO activity increased significantly according to gastric mucosal damage. Treatment with famotidine significantly decreased the rise in MPO activity, while allopurinol had no effect. The correlation between MPO activity and TBA RS was positive $(r=0.774)$.

Changes in SOD and GSH-px activities are shown in Figs. 4 and 5. SOD activity decreased significantly in gastric mucosa following gastric injury $(p<0.001)$. Treatment with famotidine and allopurinol increased the SOD activity up to the control level. The correlation coefficient between TBA RS and SOD activity was not increased $(r=-0.675)$. However, the correlation coefficient tended to be negative. On the other hand, GSH-px activity was significantly decreased in the gastric mucosa following gastric injury $(p<0.01)$, but treatment with famotidine and allopurinol could not bring the level of GSH-px activity back to the control level. There was no correlation between GSH-px and TBA RS (-0.550).

\section{Discussion}

Oxygen free radicals are regarded as mediators of acute gastric mucosal injury, particularly mucosal injury associated with ischemia-reperfusion. ${ }^{6-8,10,11,13)}$ Oxygen free radicals can attack and initiate a free radical chain reaction known as lipid peroxidation. ${ }^{9)}$ Lipid peroxidation may play an important role in the formation of gastric mucosal lesions induced by our model. First, we showed that the production of gastric mucosal injury and, TBA RS levels in the gastric mucosa increased significantly following gastric mucosal ischemia induced by clamping of the celiac artery for $30 \mathrm{~min}$ and reperfusion for $60 \mathrm{~min}$ while performing pylorus ligation for $3 \mathrm{~h}$ (Table I, Fig. 1). Many physiological processes are known to result in the production of oxygen-derived free radicals. These include enzymatic reactions, electron transport processes within the mitochondria and endoplasmic reticulum, intracellular auto-oxidation of various compounds, arachidonic acid metabolism, and the activation of phagocytic cells. However, the source of oxygen free radicals in tissues subjected to ischemia-reperfusion is not yet clear. We studied the source of oxygen free radicals. Much of the work on these sources has focused on XOD as the primary source of oxygen species produced during reperfusion. Evidence for XOD as the major source of oxygen free radicals was obtained from studies in which pretreatment was made with XOD inhibitors such as allopurinol or pterin aldehyde, ${ }^{1,6,7)}$ or following treatment with free radical scavengers and inactivation of XOD with a molybdenumfree tungstate diet. $^{8)}$ In our study, XOD activity in the gastric mucosa was increased significantly by ischemiareperfusion with pylorus ligation. Although XOD activity in the gastric mucosa was inhibited by allopurinol treatment, gastric mucosal injury did not subside and the TBA RS level in gastric mucosa did not change. These data indicate that XOD may not be a major source of oxygen free radicals in gastric mucosal injury induced by ischemia-reperfusion with pylorus ligation. According to Pitter et al., ${ }^{19)}$ in a gastric injury model induced by hemorrhagic shock in baboons, gastric lesions were not inhibited by pretreatment with allopurinol. We conclude that oxygen free radicals induced by ischemia-reperfusion were produced by a mechanism other than the XOD system.

Circulating PMN cause gastric mucosal injury by generating oxygen free radicals. ${ }^{10,11,22)} \mathrm{MPO}$, which is a marker enzyme of leucocytes, ${ }^{20-22)}$ was measured in order to examine another route of oxygen radical generation. We observed that MPO activity in the gastric mucosa was increased by ischemia-reperfusion and pylorus ligation in this experiment. MPO activity was not inhibited by allopurinol treatment, but was significantly inhibited by famotidine. A positive correlation was found between these changes in MPO activity and TBA RS level. This change in TBA RS level correlated even more closely with the index of gastric mucosal injury. Thus, the level of MPO activity was thought to represent leukocyte migration to the injured tissue. Grisham et al. ${ }^{10,11)}$ have proposed that ischemia-reperfusion results in XOD generation, superoxide-dependent accumulation of neutrofils in the intestinal mucosa, where neutrophil-derived oxidants mediate or exacerbate injury, or both. Likewise, Granger has examined the influence of ischemia-reperfusion on PMN fluxes in cat intestinal mucosa using MPO activity. ${ }^{23)} \mathrm{He}$ suggested that the oxidants generated by xanthine oxydase play a role in the recruitment of PMN. But in our result, famotidine did not inhibit XOD activity despite its inhibition of mucosal injury. And despite inhibition of XOD activity, allopurinol did not inhibit mucosal injury. Miyata and his coworker reported ${ }^{24)}$ that famotidine seemed to inhibit the decrease in gastric mucosal blood flow. It seems to inhibit sticking changes in neutrophils ${ }^{25)}$ as well as the production of a neutrophil chemotactic factor. ${ }^{26)}$ In addition, allopurinol seems not to inhibit the superoxide produced by activating purification neutrophils. ${ }^{10,27)}$ Therefore, PMN seems to play a large part in gastric mucosal injury induced by ischemiareperfusion accompanied by pylorus ligation.

We have been studying the changes in enzymes associated with the production and resolution of lipid peroxidation at inflammatory sites. ${ }^{28,29)}$ In this study, we examined

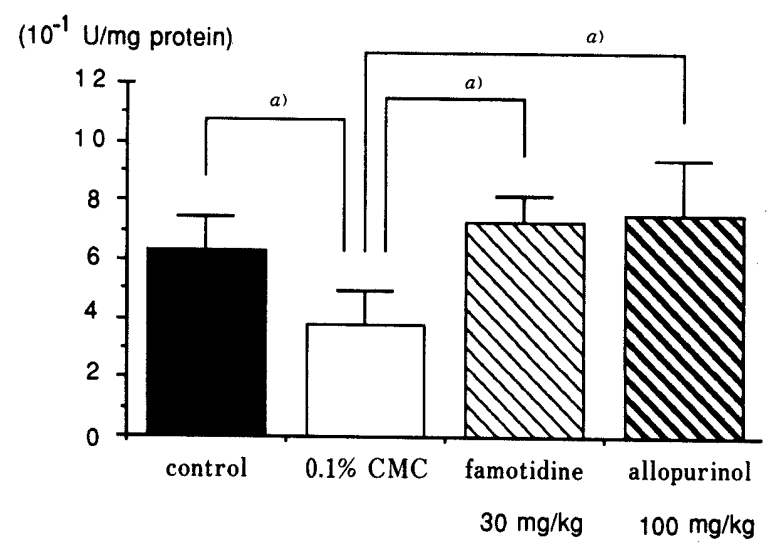

Fig. 4. Effect of Famotidine and Allopurinol on SOD Activity in the Gastric Mucosa Lesions Induced by Ischemia-Reperfusion in the Pylorus Ligated Rat

Level of SOD activity in the gastric mucosa after ischemia-reperfusion with pylorus ligation for $3 \mathrm{~h}$ in the rats. Each value is the mean \pm S.D. from $7-10$ determinations. An untreated rat was used as the control. a) $p<0.001$. 


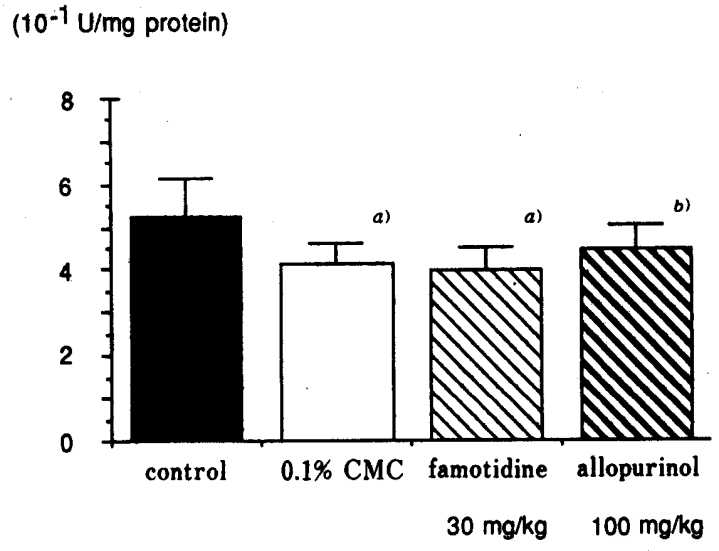

Fig. 5. Effect of Famotidine and Allopurinol on GSH-px Activity in the Gastric Mucosa Lesions Induced by Ischemia-Reperfusion in the Pylorus Ligated Rat

Levels of GSH-px activity in the gastric mucosa after ischemia-reperfusion with pylorus ligation for $3 \mathrm{~h}$ in the rats. Each value is the mean \pm S.D. from $7-10$ determinations. An untreated rat was used as the control. a) $p<0.01, b) . p<0.05$ significantly different from control.

the effects of two protective enzymes, SOD and GSH-px, against tissue peroxidative damage to the gastric mucosa. Our studies revealed that SOD and GSH-px activity significantly decreased in gastric mucosa during ischemiareperfusion in the pylorus-ligated rat (Figs. 4 and 5). Pretreatment with famotidine retarded this decrease in SOD activity level. But the decrease in GSH-px activity did not improve with any drug. Grisham et al. ${ }^{10)}$ reported that mucosal SOD and GSH decreased during ischemia and subsequent reperfusion. From this result, it was suggested that the decreases in SOD and GSH-px activity in gastric mucosa aggravated mucosal injury by free radicals and lipid peroxidation.

In conclusion, the results of this study indicate that oxygen radicals and lipid peroxidation play an important role in the pathogenesis of gastric mucosal injury induced by ischemia-reperfusion in the pylorus-ligated rat. The main source of oxygen radicals seems to be PMN. The relationship between XOD-generated superoxide and neutrophil infiltration was not considered. It is possible that neutrophil infiltration is induced by oxygen radicals produced directly from endothelial cells by ischemia. Futhermore, it also seems that lipid peroxidation is increased by the depression of SOD and GSH-px activities in gastric mucosa.

\section{References}

1) D. N. Granger, M. E. Hollwarth, and D. A. Parks, Acta Physiol. Scand., 548 (Suppl), 47 (1986).

2) K. P. Burton, J. M. McCord, and G. Ghai, Am. J. Physiol., 246, H776 (1984).

3) D. A. Parks, G. B. Bulkley, and D. N. Granger, Surgery, 94, 428 (1983).

4) D. N. Granger, D. Adkison, M. E. Hollwarth, J. N. Benoit, D. A. Parks, J. M. McCord, and P. R. Kvietys, Gastroenterology, 88, 1662 (1985).

5) M. J. Im, W-H. Shen, C. J. Pak, P. N. Manson, G. B. Bulkley, and J. E. Hoopes, Plast. Reconstr. Surg, 73, 276 (1984).

6) M. A. Perry, S. Wadhwa, D. A. Parks, W. Pickard, and D. N. Granger, Gastroenterology, 90, 362 (1986)

7) M. Itoh and P. H. Guth, Gastroenterology, 88, 1162 (1985).

8) S. M. Smith, M. B. Grisham, E. A. Manci, D. N. Granger, and P. R. Krietys, Gastroenterology, 92, 950 (1987).

9) A. L. Tappel, Fed. Proc., Fed. Am. Soc. Exp. Biol., 32, 1870 (1973).

10) M. B. Grisham, L. A. Hernandez, and D. N. Granger, Am. J. Physiol., 251 G567 (1986).

11) L. A. Hernandez, M. B. Grisham, B. Twohig, T. K. Arfors, J. Harlan, and D. N. Granger, Am. J. Physiol., 253, H699 (1987).

12) H. Sies (ed.), "Oxidative Stress," Academic Press, Inc., London, 1985.

13) T. Yoshikawa, S. Ueda, Y. Naito, S. Takahashi, H. Oyamada, Y. Morita, T. Yoneta, and M. Kondo, Free Rad. Res. Comms., 7, 285 (1989).

14) H. Ohkawa, N. Ohishi, and K. Yagi, Anal. Biochem., 95, 351 (1979).

15) P. P. Bradley, D. A. Priebat, R. D. Christensen, and G. Rothstein, J. Invest. Dermatol., 78, 206 (1982).

16) Y. Oyanagui, Anal. Biochem., 142, 290 (1984).

17) R. A. Lawrence and R. F. Burk, Biochem. Biophys. Res. Commun., 71, 952 (1976).

18) O. H. Lowry, N. J. Rosenbrough, A. L. Farr, and R. J. Randall, J. Biochem. (Tokyo), 193, 265 (1951).

19) C. V. Ritter, R. A. Hinder, M. M. J. Oosthuizen, L. G. Svensson, S. J. S. Hunter, and H. Lambrecht, Dig. Dis. Sci., 33, 857 (1988).

20) Y. Oyanagui, "Oxygen Radicals and Disease," Kagakudojinsha, Tokyo, 1989, pp. 92-94.

21) D. A. Peterson, B. Kelly, and J. M. Gerrard, Biochem. Biophys. Res. Commun., 137, 76 (1986).

22) N. R. Matheson, P. S. Wong, and J. Travis, Biochemistry, 20, 325 (1981).

23) D. N. Granger, Am. J. Physiol., 255, H1269 (1988).

24) K. Miyata, T. Kamato, A. Nishida, and K. Honda, Jpn. J. Pharmacol., 55, 211 (1991).

25) M. Suematsu, H. Nagata, Proceedings of 4th World Congress for Microcirculation, Elsevier, Amsterdam, 1987, pp. 663-666.

26) W. F. Petrone, D. K. English, K. Wong, and J. M. McCord, Proc. Natl. Acad. Sci. U.S.A., 77, 1159 (1980).

27) H. P. Jones, M. B. Grisham, S. K. Bose, V. A. Shannon, A. Schott, and J. M. McCord, Biochem. Pharmacol., 34, 3673 (1985).

28) Y. Yuda, F. Hirano, and J. Tanaka, Yakugaku Zasshi, 107, 287 (1987).

29) Y. Yuda, J. Tanaka, F. Hirano, and H. Kitagawa, Chem. Pharm. Bull., 36, 2490 (1988). 